\title{
Pull-off force of coated fine powders under small consolidation
}

\author{
Yuhua Chen, ${ }^{1}$ M. A. S. Quintanilla, ${ }^{1, *}$ Jun Yang, ${ }^{1}$ Jose M. Valverde, ${ }^{2}$ and Rajesh N. Dave ${ }^{1, \dagger}$ \\ ${ }^{1}$ New Jersey Center for Engineered Particulates (NJCEP), New Jersey Institute of Technology, \\ University Heights, Newark, New Jersey 07102-1982, USA \\ ${ }^{2}$ Faculty of Physics, University of Seville, Avenida Reina Mercedes s/n, 41012 Seville, Spain \\ (Received 11 June 2008; revised manuscript received 3 October 2008; published 14 April 2009)
}

\begin{abstract}
In this paper, a three-dimensional model taking into account the contact deformation and surface area coverage (SAC) of nanoadditives is proposed to predict the force required to separate two contacting particles (the pull-off force) under consolidation stress up to $10 \mathrm{KPa}$, for cornstarch, a Geldart group $\mathrm{C}$ powder, sparsely and densely dry-coated with nanosilica. The experimental pull-off force measurement is conducted in a Seville powder tester. Comparison of the predicted results with the experimental results indicates (1) that the pull-off force of sparsely coated cornstarch is larger than that of densely coated cornstarch due to the greater hardness and small particle radius of fumed silica; (2) there is not a continuous variation in the pull-off force with the coverage of silica; on the contrary, values of the pull-off force of sparsely coated samples are grouped in similar range, while the values of the pull-off force of densely coated samples are grouped in another range of lower values. (3) Within a range, the SAC does not have a big effect on the pull-off force for sparsely coated samples and only a slight effect for densely coated samples (4) the pull-off force increases with increasing consolidation force due to larger deformation in the contact area; (5) under consolidation stresses up to $10 \mathrm{KPa}$, the deformation of the cornstarch particles is not large enough to fully embed the nanosized silica.
\end{abstract}

DOI: 10.1103/PhysRevE.79.041305

PACS number(s): 83.80.Fg, 81.05.Rm, 81.20.Ev

\section{INTRODUCTION}

Fine particles classified as Geldart group C powders [1] have gained tremendous interest from academia and industry due to their promising applications in chemical, pharmaceutical, and cosmetics industries. It is well known that fine powders have very poor flowability due to the dominant interparticle adhesion force, mainly attributed to van der Waals forces for dry and neutral particles [2]. Interparticle adhesion force can easily cause arches inside the powder beds and hinder particles from flowing. Dry particle coating [3] is an efficient technique to prepare engineered particles and it helps reduce the adhesion force of very fine powders and improve their flowability and fluidizability $[4,5]$ by depositing nanosized particles on the surface of primary cohesive particles to alter the surface morphology and roughness. However, at present, the mechanism of how nanoadditives improve fluidizability is not fully understood. Most researchers believe that the fine additives act as spacer particles [6-8], while some believe that there is a "ball bearing" or lubricant effect $[9,10]$ and some have even proposed that nanoadditives may cause neutralization of electrostatic charges [11].

Adhesion force between contact particles has been investigated for several decades. A number of models have been developed to calculate the adhesion force between smooth surfaces undergoing elastic deformation such as JohnsonKendall-Roberts (JKR) model [12], Deryaguin-MullerToporov (DMT) model [13], and their extended and modified models [14]. However, the measured adhesion force for par-

\footnotetext{
*Present address: Faculty of Physics, University of Seville, Avenida Reina Mercedes s/n, 41012 Seville, Spain.

†Corresponding author, dave@njit.edu
}

ticles between $10-100 \mu \mathrm{m}$ is always less than the theoretical results due to the surface roughness which reduces the contact area between two contiguous bodies with respect to the case when contacting surfaces are perfectly smooth [15]. Yang et al. [4], Rumpf [7], Xie [8], and Mei et al. [16] tried to take into account the effect of surface roughness and stated that the adhesion force between two rough spheres is composed of the attraction between two parent spheres representing the large scale curvature of the surfaces and the adhesion between an asperity and the parent spheres. They found out that asperities can dramatically reduce the interparticle adhesion force approximately by the factor of $d_{p} / d_{a}$, here $d_{p}$ is the size of parent particles and $d_{a}$ is the asperity size. Nevertheless, in those models particles are considered as rigid bodies and no surface deformation was included. Rabinovic et al. [17,18] and Li et al. [19] proposed models accounting for the elastic deformation of the asperities based on the DMT and JKR models and compared their calculation with atomic force microscopy (AFM) measurements.

Tomas [20] pointed out that the load force at which a contact starts to suffer plastic deformation decreases with particle size, so fine particles and asperities are easily deformed elastoplastically even under the effect of interparticle adhesion force alone. Castellanos [21] estimated the critical asperity size to have plastic deformation under the action of only the interparticle adhesion force (that is, under zero external load) and concluded that for asperities less than $0.1 \mu \mathrm{m}$ the contact area would yield plastically. Castellanos et al. [22-24] measured the tensile strength defined as the force required to break a bulk powder bed of fine toner particles blended with nanoadditives under small consolidation. They found a sublinear increase in the tensile strength with consolidation stress compatible with the elastic-plastic deformation. Restagno et al. [25] also observed that the pull-off force was not solely determined by the surface properties but also depends on the maximum normal load $F_{\max }$ that had 
been applied on the surfaces before pulling them apart.

Since particles are compressed at least by the weight of particles above them when stored, transported and processed, it is important to account for the plastic deformation at such low consolidations when proposing a mechanism of the improvement in fluidizability or flowability by using dry coating process. However, the elastoplastic deformation is too complicated to be calculated analytically. The solution of this problem has been provided by numerical methods recently [26,27]. Instead of using numerical simulation, Johnson [28] derived a pull-off force model between a rigid flat surface and a deformable sphere assuming that deformation is in fully plastic regime and the pressure distribution within contact area is similar to the Hertzian pressure profile during unloading. The pull of force can be calculated by

$$
F_{\mathrm{pf}}=\frac{3}{2} \pi \Delta \gamma \frac{4 E^{*} F_{o n}^{1 / 2}}{3(\pi H)^{3 / 2}} .
$$

Here, $\Delta \gamma$ is the work of adhesion between two solid surfaces $E^{*}=\left[\left(1-\nu_{1}^{2}\right) / E_{1}+\left(1-\nu_{2}^{2}\right) / E_{2}\right]^{-1}, H$ is the hardness of the soft material, and $F_{\text {on }}$ is the compressive force between surfaces in contact.

Based on Johnson's assumption, Maugis and Pollock [29] suggested to substitute $F_{o n}$ with $F_{o n}+2 \pi \Delta \gamma R^{*}$ to include the effect of the initial adhesion force when deriving the pull-off force,

$$
F_{\mathrm{pf}}=\frac{3}{2} \pi \Delta \gamma \frac{4 E^{*}\left(F_{o n}+2 \pi \Delta \gamma R^{*}\right)^{1 / 2}}{3(\pi H)^{3 / 2}} .
$$

Here, $R^{*}$ is the reduced radius of curvature of the contact surfaces

$$
\frac{1}{R^{*}}=\frac{1}{R_{1}} \pm \frac{1}{R_{2}},
$$

where $R_{1}$ and $R_{2}$ are the radius of two contacting spheres (in this case, the plus symbol is used) or $R_{1}$ is the radius of a sphere in a concavity of radius $R_{2}$ (in this case, the symbol minus is used).

Podczeck and Newton [15] also derived a similar equation as Johnson's and estimated the true contact area between rough particles and a flat surface in contact. Recently Quintanilla et al. [30] applied a modified Maugis-Pollock equation to predict the pull-off force between fine toner particles blended with fumed silica nanoparticles and compared the predicted force with experimental measurements. Values of the additive concentration separating the case of surface contact between toner and toner, between toner and silica, and between silica and silica were proposed.

These models only considered the contact between a single asperity (additive) and a flat surface. However, the actual contact between two rough surfaces is likely to involve a number of asperities. For reasons of stability of the contact against tangential forces and torques acting on the contact, we will assume that a typical contact is formed by three asperities (additives) with a relatively flat surface. In this paper, a pull-off force model is proposed taking into account the effect of surface area coverage of nanoparticles and fully plastic contact deformation under small consolida-

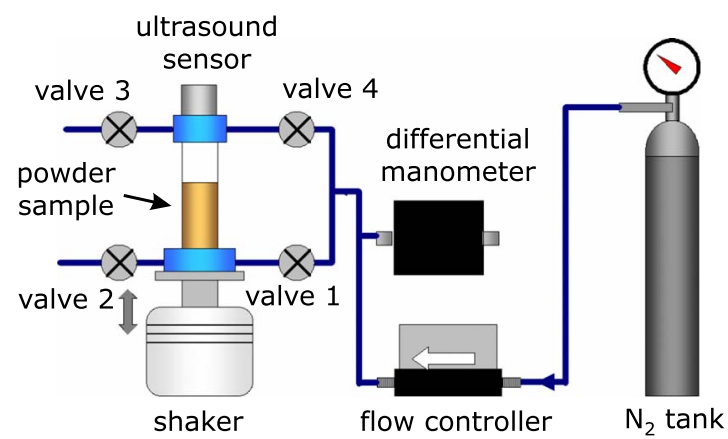

FIG. 1. (Color online) Sketch of Seville powder tester.

tion for dry-coated fine particles. The model is used to predict the values of the tensile strength of a set of powders measured in a Seville powder tester (SPT) [31].

\section{EXPERIMENTAL}

\section{A. Seville powder tester}

The Seville powder tester [22,31] based on the use of a fluidized bed can achieve a reproducible initial state of tested material removing the memory or history of cohesive powders by fluidizing the powder in the bubbling regime. Our test unit uses a fluidized bed with a gas distributor located on the bottom of a polycarbonate column and an ultrasonic sensor fitted on the top to measure the height of powder bed, which can be converted to the solid fraction of powder bed (see Fig. 1). Four valves are employed to control the direction of gas flow; with valves 1 and 3 opening the gas flow upward to break or fluidize the powder, whereas valves 2 and 4 opening the gas flow downward to compress the powder with desired consolidation stress. A differential pressure transducer and a digital flow controller are utilized to measure the pressure drop across the powder bed and control the gas flow rate through the bed, respectively. In order to measure the tensile strength and solid fraction as a function of consolidation stress, the tested powder is initially fluidized in the bubbling regime to get rid of the memory of its initial heterogeneous state. Then the fluidized powder bed settles down by either turning off the gas flow for overweight consolidation or maintaining a specified upward gas flow to perform underweight consolidation. In order to carry out the overweight consolidation, the downward gas flow is increased slowly to a desired value. This results in a homogenously distributed pressure on the powder, which is pressed against the distributor plate. The consolidation stress of overweight consolidation at the bottom of powder bed is the pressure drop plus the powder weight per unit area,

$$
\sigma_{c}=\Delta p+\frac{m g}{A} .
$$

Here, $\sigma_{c}$ is the consolidation stress, $\Delta p$ is the pressure drop across the powder bed, $m$ is the powder mass, and $A$ is crosssection area of the plastic column.

For underweight consolidation, due to the release of powder weight by upward gas flow, the consolidation stress is the powder weight per unit area reduced by the pressure drop, 
TABLE I. Material properties of guest and host powders.

\begin{tabular}{|c|c|c|c|c|c|c|c|c|}
\hline & Material & Particle size & $\begin{array}{l}\text { Young's modulus } \\
(\mathrm{GPa})\end{array}$ & Poisson ratio & $\begin{array}{l}\text { Hamaker constant } \\
\qquad\left(10^{-20} \mathrm{~J}\right)\end{array}$ & $\begin{array}{l}\text { Surface energy } \\
\qquad\left(\mathrm{J} / \mathrm{m}^{2}\right)\end{array}$ & $\begin{array}{l}\text { Hardness } \\
\text { (GPa) }\end{array}$ & $\begin{array}{l}\text { Yield stress } \\
\quad(\mathrm{MPa})\end{array}$ \\
\hline Guest & R972 & $16 \mathrm{~nm}$ & 74 & 0.17 & 7.3 & $0.025^{\mathrm{a}}$ & $6^{\mathrm{b}}$ & $1100^{\mathrm{b}}$ \\
\hline Host & cornstarch & $15 \mu \mathrm{m}$ & $9.4^{\mathrm{c}}$ & 0.33 & 20 & $0.5^{\mathrm{d}}$ & 0.93 & $344^{\mathrm{c}}$ \\
\hline
\end{tabular}

${ }^{\mathrm{a}}$ Reference [38].

${ }^{\mathrm{b}}$ Reference [43].

${ }^{\mathrm{c}}$ Reference [42].

${ }^{\mathrm{d}}$ Reference [36].

$$
\sigma_{c}=\Delta p-\frac{m g}{A}
$$

Subsequently, the upward gas flow is increased gradually to break the powder bed. With increasing gas flow rate, the pressure drop through the powder bed initially increases linearly because the bed is unperturbed. The pressure drop balances the powder weight per unit area $m g / A$ at the incipient fluidization velocity and the powder bed begins to be under tension. Further increasing the gas flow rate will make the pressure drop increase till at some point the powder bed breaks at the bottom [31]. Therefore, the tensile strength of the powder is determined by the overshoot of pressure drop across the powder bed over the powder weight per unit area,

$$
\sigma_{t}=(\Delta p)_{\max }-\frac{m g}{A} .
$$

It is noted that the bed height cannot be larger than the bed diameter in order to minimize the wall effect on measurement.

\section{B. Materials}

We have measured the tensile strength of five cornstarch samples coated with different level of fumed silica R972 by the dry coating process conducted in magnetically assisted impaction coater (MAIC) [3-5]. Cornstarch is a typical cohesive powder with nominal size of $15 \mu \mathrm{m}$, while fumed silica R972 is a nanosized powder with size of $16 \mathrm{~nm}$. Properties of guest (silica) and host (cornstarch) particles are listed in Table I and more detailed properties of host and guest powders could be found in Ref. [5]. Figure 2 shows scanning electron microscopy (SEM) images of cornstarch coated with different levels of fumed silica R972. These SEM images elucidate that guest particles are evenly coated onto the host particle surface (white spots are nanosilica particles while the gray background is the cornstarch surface). Small silica agglomerates can be identified on the cornstarch surface because sintered silica chains are not completely broken by the dry coating process. However, the particles in an aggregate arrange themselves to be in contact with the cornstarch surfaces rather than forming layers on top of each others, so even inside an aggregate the local asperities are formed by silica nanoparticles.

\section{Transition between guest-host contact and guest-guest contact}

Contact between two dry-coated fine particles can be established through host and host surfaces, guest and host surface, and between guest particles depending on the surface area coverage $(\mathrm{SAC})$ of the guest particles. The $C_{S A}$ is defined as the ratio of the total projected area of the guest particles to the surface area of the host particle to which the guest particles are attached assuming that the guest particles are evenly and individually distributed on the host particle surface. The geometry of each one of these types of contacts is shown in Fig. 3. The adhesion forces for these three different contact modes are different due to different roughness, material properties, and contact deformation. The expression for the SAC is as follows:

$$
C_{S A}=\frac{\frac{1}{4} N \pi d^{2}}{\pi D^{2}} \times 100 \%=\frac{W_{t} D^{3} \rho_{D}}{d^{3} \rho_{d}} \frac{d^{2}}{4 D^{2}} \times 100 \% .
$$

Here $N$ is the number of guest particles coated on a single host particle, $d$ is the diameter and $\rho_{d}$ is the density of the guest particle, $D$ is the diameter and $\rho_{D}$ is the density of the host particle, and $W_{t}$ is the weight ratio of guest particle to the host particle.

In a guest-host contact, the distance $L_{0}$ between two host particles is determined by the guest particle size $d$, host particle size $D$, and the SAC [5],

$$
L_{0}=\sqrt{(d+D)^{2}-\frac{1.21}{C_{S A}} d^{2}}-D .
$$

The critical SAC between host-host contact and guest-host contact is the SAC that eliminates the direct contact between host particles and it is found setting $L_{0}$ in Eq. (8) to be zero,

$$
C_{S A}^{\text {guest-host }}=\frac{1.21}{1+2(D / d)} \times 100 \% .
$$

For cornstarch-fumed silica R972, $d=16 \mathrm{~nm}$ and $D$ $=15 \mu \mathrm{m}$ and the critical surface area coverage for the transition to guest-host contact is $C_{S A}^{\text {guest-host }}=0.06 \%$ corresponding to a weight ratio of guest particle to host particle of $0.0005 \%$ using $\rho_{d}=2.65 \mathrm{~g} / \mathrm{cm}^{3}$ and $\rho_{D}=1.55 \mathrm{~g} / \mathrm{cm}^{3}$. These values represent the ideal values of the SAC and the weight ratio at which we could expect to have a majority of guesthost contacts. They would be valid if the surface of the particles was perfectly smooth. However, in the presence of 


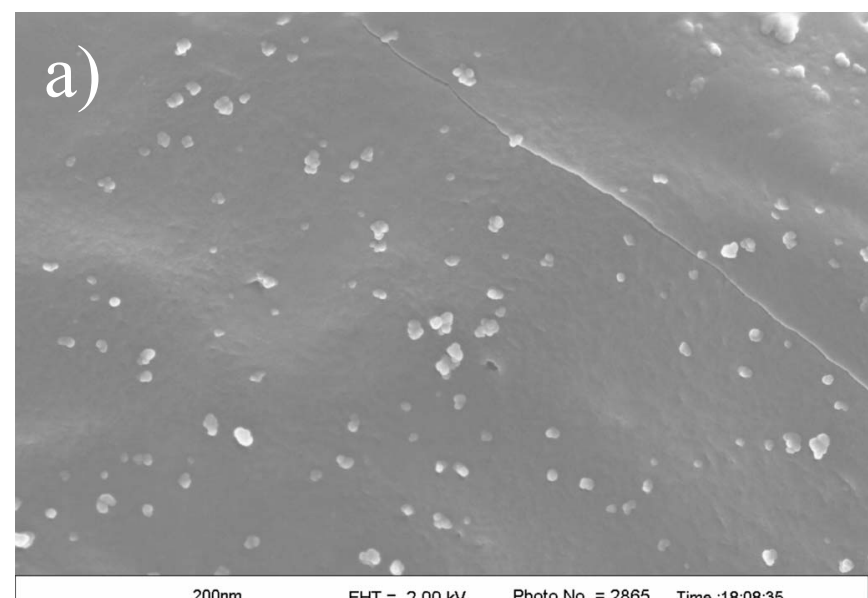

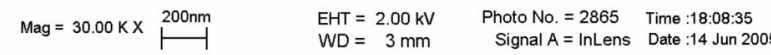

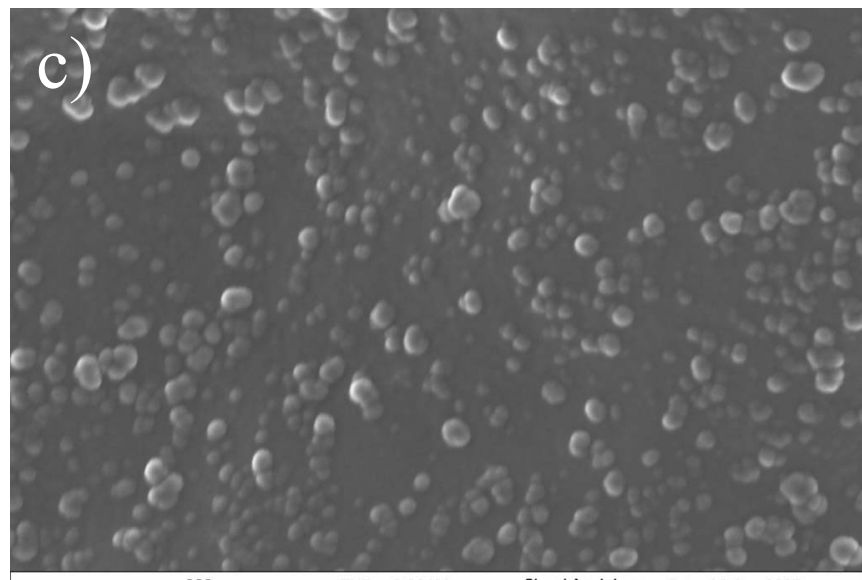

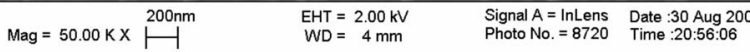

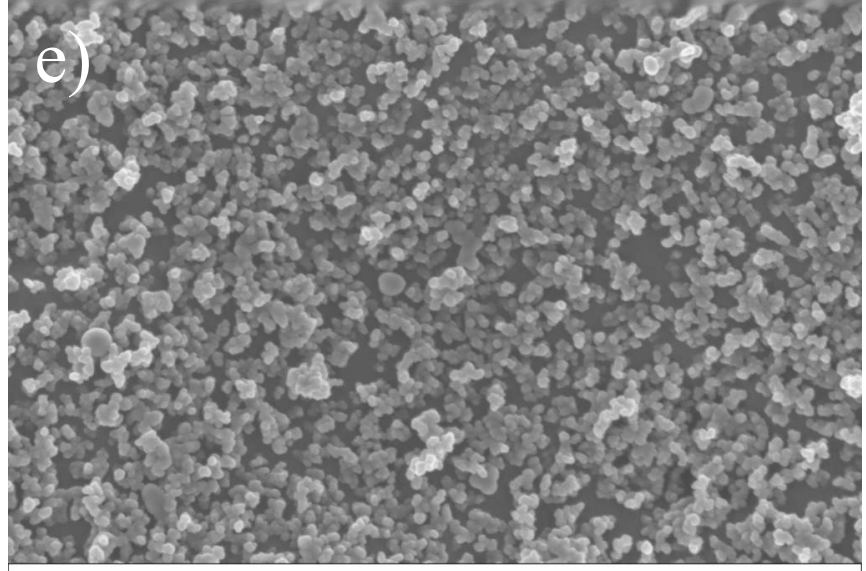

$\mathrm{Mag}=30.00 \mathrm{Kx} \stackrel{200 \mathrm{~nm}}{\longmapsto}$

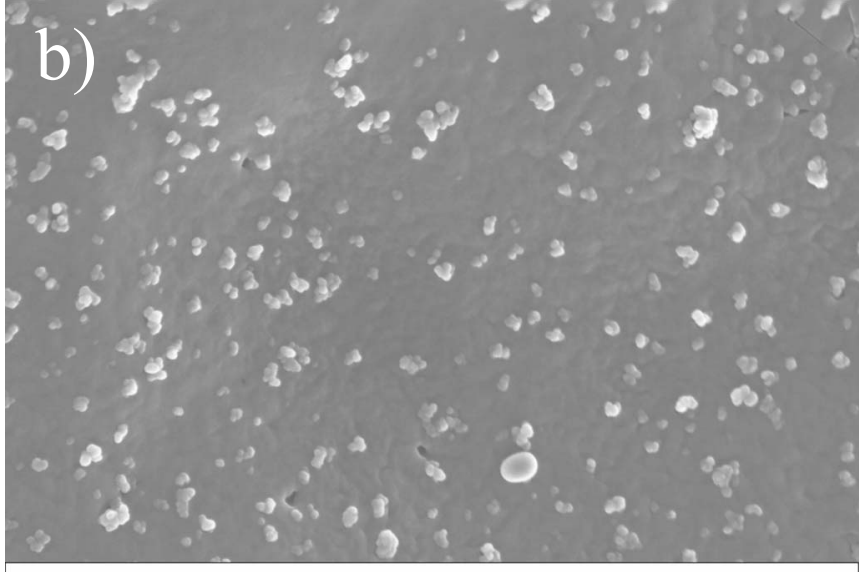

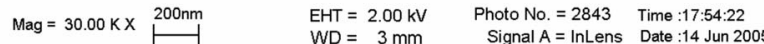

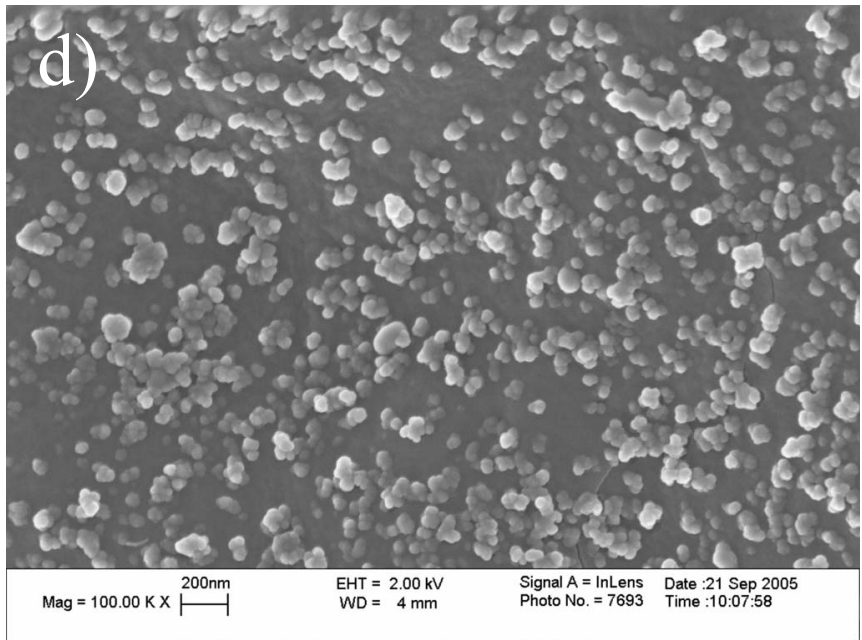

FIG. 2. SEM images of coated cornstarch with guest weight ratio of (a) $0.05 \%$, (b) $0.1 \%$, (c) $0.2 \%$, (d) $0.5 \%$, and (e) $1.0 \%$.

asperities, the value of the asperity size should be substituted in $D$ instead of the host particle diameter to obtain a more realistic value of the $\mathrm{SAC}$ yielding $C_{S A}^{\text {guest-host }}=0.96 \%$ with a corresponding weight ratio of approximately $0.01 \%$ if the asperity size of cornstarch is taken as $1 \mu \mathrm{m}$ based in estimations from SEM images [5].
At the value of the SAC for the transition from guest-host contact to guest-guest contact, three guest particles on one host particle surface are tangent to the guest particle on the other host particle surface as depicted in Fig. 4(c). The value of the SAC is given by the ratio of the shaded area to the area of the triangle $C_{2} C_{3} C_{4}$, 


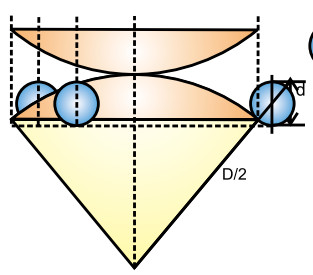

a)

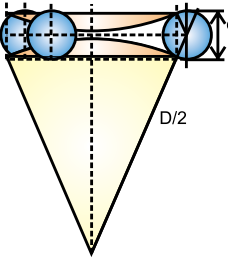

b)

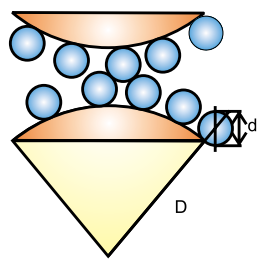

c)
FIG. 3. (Color online) Contact between two dry-coated fine particles depending on the different SAC; (a) host-host contact, (b) guest-host contact, and (c) guest-guest contact.

$$
\begin{gathered}
C_{S A}^{\text {guest-guest }}=\frac{3 \times \frac{\pi}{6}\left(\frac{d}{2}\right)^{2}}{\frac{3 \sqrt{3}}{4} d^{2}} \times 100 \% \Rightarrow \\
C_{S A}^{\text {guest-guest }}=\frac{\pi}{6 \sqrt{3}} \times 100 \% \simeq 30 \%,
\end{gathered}
$$

corresponding to a transition weight ratio of approximately $0.22 \%$; as it can be seen in Eq. (10), the value of $C_{S A}^{\text {guest-guest }}$ is only determined by the contact geometry without the dependence of the guest particle size and host particle size.

\section{Definition of sparse and dense coatings}

In this paper, we will say a coating is sparse when the guest-host contacts are the most common contact type. Direct host-host contacts are not considered since the weight ratio of guest particles required for them to be the most numerous contact type is too small to be practical. As described in Ref. [5], in a guest-host contact the pull-off force defined as the particle-particle adhesion force at the moment when two particles are separated consists of the adhesion between host and guest particles and the attraction between host-host particles. A coating is dense when guest-guest contacts are the most frequent type. As shown in Figs. 3(c) and 4, in this type of contact ideally one guest particle on a host particle contacts directly with three guest particles on another host particle.

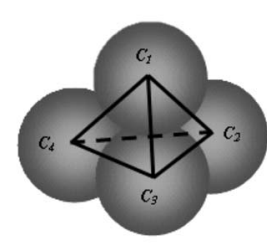

a)

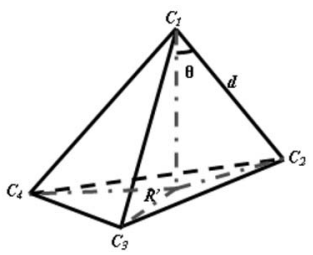

b)

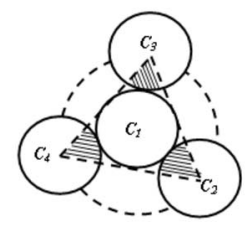

c)
FIG. 4. (a) Sketch of guest-guest contact, (b) three-dimensional plot, and (c) projected plot of critical guest-guest contact; $R^{\prime}$ is the circum radius of triangle $C_{2} C_{3} C_{4}$ formed by three guest particles on the same host particle; $C_{2}, C_{3}$, and $C_{4}$ are the centers of the guest particles on the same host particle. $d_{g}$ is the distance between centers of guest particles on different host particles.

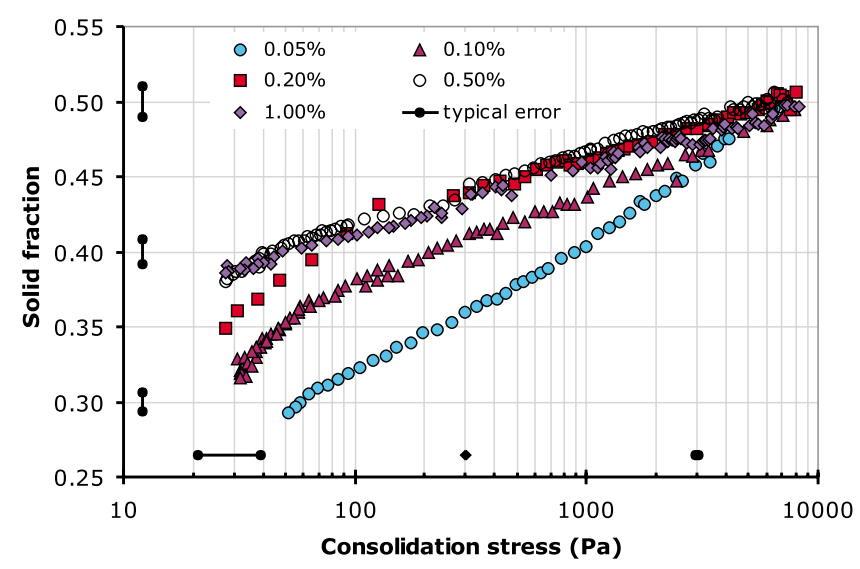

FIG. 5. (Color online) Solid fraction in powder bed as a function of consolidation stress for different weight percentages of silica R972. Horizontal black bars show the error in the consolidation stress for cornstarch $0.1 \%$ at 30,300 , and $3000 \mathrm{~Pa}$ of consolidation; whereas vertical bars show the error in the solid fraction for cornstarch $0.1 \%$ at solid fractions $\phi=0.30,0.40$, and 0.50 . Typical errors for the other materials are comparable to those of cornstarch $0.1 \%$.

\section{E. Experimental results}

\section{Solid fraction}

Figure 5 plots the experimental results of the solid fraction in the powder bed as a function of consolidation stress $\sigma_{c}$ (for $\sigma_{c}$ less than $10 \mathrm{KPa}$ ) for both sparsely and densely coated cornstarch with different silica weight ratios, corresponding to different SAC. Initially, larger SAC leads to higher solid fraction or higher packing density indicating less cohesiveness of powders. Powders become more and more densely packed with the increase in consolidation stress. In the case of sparse coating, it is seen that for a given consolidation stress the solid fraction has a larger value for a higher SAC. On the other hand, the SAC only has slight effect on the solid fraction in the case of dense coating. With increasing the consolidation stress, the difference of solid fraction between coated cornstarch with different additive level decreases probably due to the completion of particle rearrangement. The increase in contact numbers between dry-coated particles raises the difficulty of particle rearrangement and particles tend to deform.

\section{Tensile strength}

Experimental tensile strength for both sparsely coated and densely coated cornstarch as a function of consolidation stress is shown in Fig. 6. It is seen that the relationship between tensile strength and consolidation stress is nonlinear which can be explained if the deformation on the contact area between dry-coated particles is plastic [30]. It is clearly seen that densely coated cornstarch particles have less tensile strength than sparsely coated cornstarch particles, indicating less cohesiveness and better flow and fluidization behaviors. However, within each coating group (sparse or dense coating), tensile strength of coated samples with different SACs measured by SPT have approximately similar values, indicating that SAC does not play important role on the pull-off force. This fact suggests that while the tensile strength of 


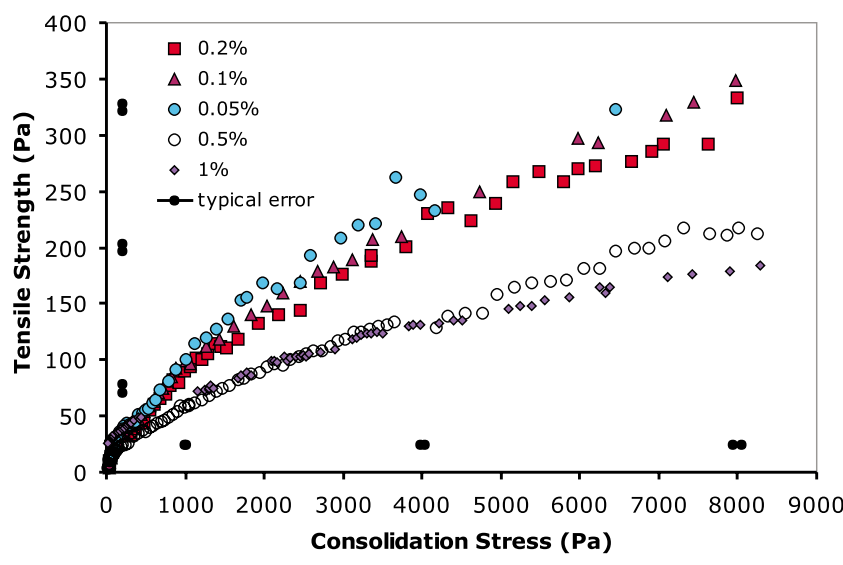

FIG. 6. (Color online) Experimental tensile strength as a function of consolidation stress for different weight percentage of silica R972. Horizontal black bars show the error in the consolidation stress for cornstarch $0.1 \%$ at 1,4 , and $8 \mathrm{KPa}$ of consolidation; whereas vertical bars show the error in the tensile strength for cornstarch $0.1 \%$. Typical errors for the other materials are comparable to those of cornstarch $0.1 \%$.

coated fine particles is significantly affected by the coating type, (e.g., sparse coating or dense coating), it is only slightly affected by differences in coating levels (e.g., surface area coverage or weight ratio of guest particles) within a specific coating coverage type.

\section{THEORETICAL}

\section{A. Pull-off force for sparsely coated fine particles}

Maugis and Pollock [29] investigated the pull-off force between a rigid particle and a flat surface under the elastoplastic deformation and proposed three adherence modes depending on the separation behaviors of particles. The contact between a host and a guest particles falls in the case of brittle separation following elastoplastic and fully plastic deformation. In this case, Maugis and Pollock assumed that the true pressure distribution in the contact area is similar to the Hertzian distribution and the contact area retains its shape and size during unloading. Thus, this problem is analogous to the elastic contact between a sphere of radius $R_{1}$ and a spherical concavity of radius $R_{2}$ (as shown in Fig. 7). The resulting pull-off radius $R$ can be calculated as follows:

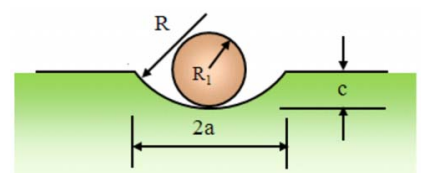

a)

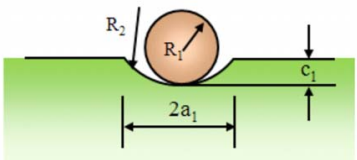

b)
FIG. 7. (Color online) Deformation of the contact area (a) during unloading and (b) after separation. $R$ is the pull-off radius, $R_{1}$ is the guest particle radius, $R_{2}$ is the indentation radius after separation, $a$ and $a_{1}$ are the contact area radius during and after unloading, $c_{1}$ is the center approach between guest and host particles after unloading, and $c$ is the center approach between guest and host particles during unloading.

$$
\begin{aligned}
& \frac{1}{R}=\frac{1}{R_{1}}-\frac{1}{R_{2}}, \\
& R=\frac{4 E^{*} F^{1 / 2}}{3(\pi H)^{3 / 2}} .
\end{aligned}
$$

The pull-off force is given by

$$
F_{\mathrm{pf}}=\frac{4}{3} \lambda \pi \Delta \gamma \frac{E^{*} F^{1 / 2}}{(\pi H)^{3 / 2}} .
$$

Here, $\lambda$ is a constant depending on the Tabor [32] parameter [if $\nu=\left(R \Delta \gamma^{2} / E^{* 2} z_{0}^{3}\right)^{1 / 3} \gg 1, \lambda$ tends to $3 / 2$; if $\nu \ll 1, \lambda$ tends to 2] and $F$ is the contact load which is the sum of the contributions of the interparticle compressive force and the surface forces,

$$
F=F_{\text {on }}+2 \pi \Delta \gamma R^{*},
$$

where $F_{\text {on }}$ is the interparticle compressive force compressing the particles and $R^{*}$ is the reduced radius of curvature of the contact surfaces. For dry-coated fine particles, since the typical guest particle $(<50 \mathrm{~nm})$ is much smaller than the typical asperity $(200 \mathrm{~nm})$ [33] or even the roughness observed from the SEM images, the host surface can be considered as a flat deformable surface and the guest particle as a rigid sphere. The interparticle compressive force $F_{\text {on }}$ is assumed to be evenly distributed onto the three contact points at which a guest particle contacts with the host particle, so the actual contact load on each guest particle is

$$
F_{c}=\frac{1}{3} F_{o n}+2 \pi \Delta \gamma R^{*}
$$

Pull-off force at each contact point can be calculated substituting $F$ in Eq. (13) with $F_{c}$ in Eq. (15).

Due to the elastic-plastic deformation between the host and guest particles, the distance between two host particles decreases. The center approach $c$ between the guest and host particles when separation happens is calculated by

$$
c=R-\sqrt{R^{2}-a^{2}} .
$$

$R$ is calculated from Eq. (12) and $a=\sqrt{F / \pi H}$ is the radius of contact area between the guest and host particle surfaces in contact. So, the adhesion force between two host particles is

$$
F_{h h}=\frac{A D_{\text {asperity }}}{24 L^{2}}
$$

Here, $L \simeq L_{0}-2 c$ is the distance between the host particles, $L_{0}$ is the initial distance calculated through Eq. (8), $A$ is the Hamaker constant, and $D_{\text {asperity }}$ is the asperity size on the host particle surface.

The total pull-off force for sparsely coated fine particles is the combination of the pull-off force between three guest particles and a host particle with the host-host attractive force at the separation

$$
F_{a d}=4 \lambda \pi \Delta \gamma \frac{E^{*} F_{c}^{1 / 2}}{(\pi H)^{3 / 2}}+\frac{A D_{\text {asperity }}}{24 L^{2}} .
$$




\section{B. Pull-off force for densely coated fine particles}

As it has been said, for densely coated particles only guest particles are in contact with each other. Since we have made the assumption that guest particles are smooth and spherical, the elastoplastic contact between two guest particles can be described by existing models of elastoplastic contact between spheres. In the model of Thornton and Ning [34] for the collision behavior of adhesive elastic-plastic spheres, a JKR pressure distribution is assumed to exist over the contact area with a cutoff corresponding to the limiting contact pressure during the loading stage. Thomas [20] balanced attractive forces with elastic and plastic repulsive forces and derived his adhesion force model based on Thornton and Ning's model. Mesarovic and Fleck [35] simulated the elastic-plastic loading stage between two adhesive elasticplastic spheres and found that the pressure distribution over the contact area at the end of loading is approximately uniform for elastic-perfectly plastic materials. A pull-off model was also proposed by Mesarovic and Johnson [36] based on the assumption that during unloading the deformation is predominantly elastic. This model yields a slightly different result for the pull-off force and the pull-off radius than those obtained by Johnson,

$$
R=\frac{R^{*} a^{\prime}}{p} .
$$

Here, $a^{\prime}=\sqrt{F /(\pi p)}$ is the radius of the contact area at the end of loading and $p$ is the average pressure over the contact area. The average contact pressure $p$ is equal to the hardness $H$ in the fully plastic similar regime; that is, as long as the contact area radius is much smaller than the radius of the contacting spheres [35].

Under small consolidation, in order to simplify the calculation, it is assumed that the deformation between guest particles is much smaller than the guest particle size, contact points on the same guest particle are independently deformed, and no coalescence of deformed spots occurs. Under these assumptions, the effective pull-off radius could be derived according to Mesarovic and Johnson [36],

$$
R=\frac{\pi E^{*} F^{1 / 2}}{(\pi H)^{3 / 2}}
$$

and the resulting adhesion between two guest particles is

$$
F_{\mathrm{pf}}=\lambda \pi \Delta \gamma R=\lambda \Delta \gamma \pi^{2} \frac{E^{*} F_{c}^{1 / 2}}{(\pi H)^{3 / 2}} .
$$

As shown in Fig. 4, only by considering a frictionless contact, the interparticle compressive force exerted on densely coated particles may be evenly distributed on to three contact spots between guest particles. So the normal contact load $F_{c}$ is calculated by

$$
F_{c}=\frac{F_{\text {on }}}{3 \cos \theta}+2 \pi \Delta \gamma R^{*} .
$$

Here, $\cos \theta=\sqrt{1-R^{\prime 2} / d^{2}}=\sqrt{1-0.302 / C_{S A}}$ since SAC is a function of the guest and host particle size. $R^{\prime}$ is the circum radius of the triangle formed by three guest particles as shown in Fig. 4.

On the other hand, when the two host particles are pulled apart, only a fraction $\cos \theta$ of the pull-off force $F_{\text {pull-off }}$ acts in the normal to the contact area, therefore

$$
F_{a d}=\frac{3 F_{\mathrm{pf}}}{\cos \theta}=\frac{3 \lambda \Delta \gamma \pi^{2}}{\cos \theta} \frac{E^{*} F_{c}^{1 / 2}}{(\pi H)^{3 / 2}},
$$

where we have neglected the attractive forces between host particles since, giving that the two host surfaces are separated by approximately two guest particle diameters, their contribution to the pull-off force is much smaller than the contact adhesion between guest particles, as it will be further discussed in Secs. IV A-IV D.

\section{RESULTS AND DISCUSSIONS}

As seen in Secs. II A and III B where we have presented the model, the interparticle pull-off force is a function of work of adhesion, elastic modulus of contact materials, hardness of contact materials, the guest particle size, the coating type as well as the interparticle compressive force. In this section, we will present the predictions of the model for the materials described in Sec. II E using the values listed in Table I for the input parameters and then we will discuss the relative importance of the indeterminacy of those parameters in the results of the model (except the guest particle size, which we consider to be well characterized by the manufacturer).

\section{A. Correlation between bulk stresses and interparticle forces}

The consolidation stress and tensile stresses measured in the SPT have to be converted into interparticle contact force to calculate the pull-off force using proposed model [Eqs. (18) and (23)]. We have used Rumpf's Eq. (24),

$$
F_{i}=\sigma_{i} d_{p}^{2} \frac{2(1-\phi)^{3 / 2}}{\phi} .
$$

Here, $F_{i}$ is the interparticle contact force, $\sigma_{i}$ is the bulk stress, $d_{p}$ is the particle size, and $\phi$ is the solid fraction in the powder bed. When using Eqs. (15) and (22) to evaluate the contact force, if the consolidation stress (alt tensile strength) is used in $\sigma_{i}$ when applying Eq. (24), $F_{i}$ corresponds to the load force (alt pull-off force).

Figure 8 shows how the interparticle pull-off force is calculated and how its comparison with experimental results is achieved. In order to compare the analytical tensile strength with the experimental one, the experimental consolidation stress and solid fraction are employed to determine the interparticle compressive force between two dry-coated particles by using modified Rumpf's Eq. (24). Then the interparticle contact force is calculated by adding the surface force to the interparticle compressive force according to Maugis and Pollock's argument [29]. The predicted values of the tensile strength are found using Eq. (18) or Eq. (23) to calculate the pull-off forces for each interparticle contact force. Finally, the interparticle pull-off force is converted into the tensile strength by employing again the modified Rumpf's Eq. (24). 


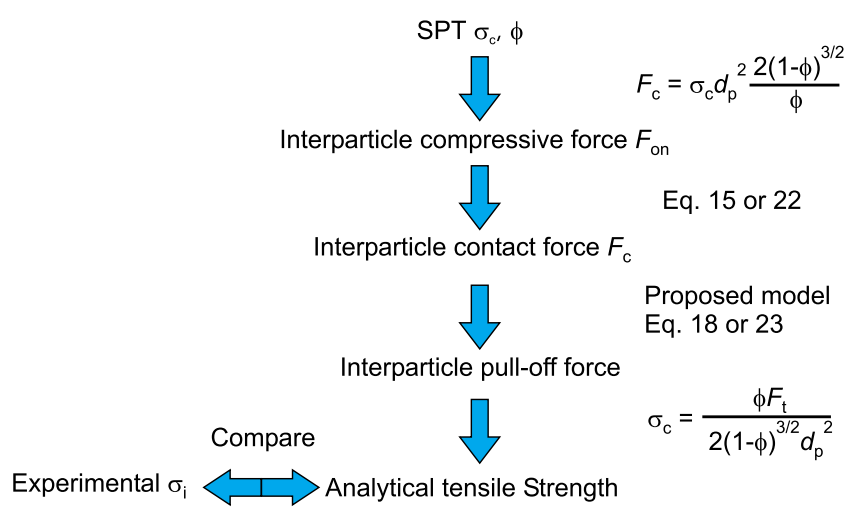

FIG. 8. (Color online) Flow chart of force calculation and comparison.

\section{B. Results for sparsely coated cornstarch}

The relationship between interparticle compressive force and consolidation stress for cornstarch with different SAC is displayed in Fig. 9. This plot illustrates that for consolidation less than $4000 \mathrm{~Pa}$, the less-coated cornstarch has larger interparticle compressive force for a given consolidation stress due to having a smaller solid fraction caused by the more intensive cohesiveness. On the other hand, with consolidation stress greater than $4000 \mathrm{~Pa}$, in spite of a slight difference, the interparticle compressive forces approximately have the same value at given consolidation stress for all sparsely coated samples since the solid fraction becomes similar and powders have the same size. It is also worthy to remind that in Eq. (15), the actual contact force between a guest particle and host particle surface is the sum of one third of interparticle compressive force and the initial adhesion force $2 \pi \Delta \gamma R^{*}$ between guest particle and host particle surface. In view of Fig. 9, the initial adhesion force can be neglected for consolidation stress greater than $100 \mathrm{~Pa}$ for our

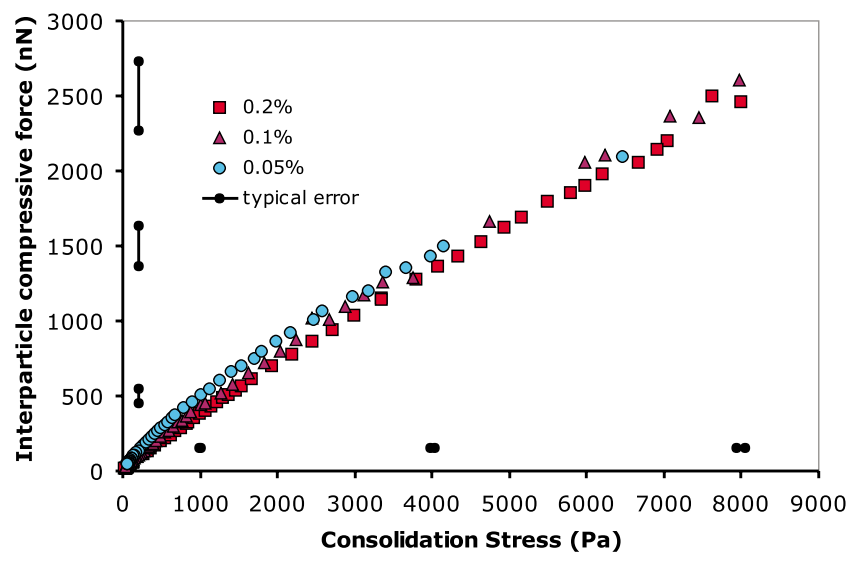

FIG. 9. (Color online) Interparticle compressive force as a function of the different consolidation stress for different weight ratio of nanoadditives. Horizontal black bars show the error in the consolidation stress for cornstarch $0.1 \%$ at 1,5 , and $9 \mathrm{KPa}$ of consolidation; whereas vertical bars show the error in the interparticle compressive force estimated from the consolidation stress using Eq. (22) for cornstarch $0.1 \%$. Typical errors for the other materials are comparable to those of cornstarch $0.1 \%$.

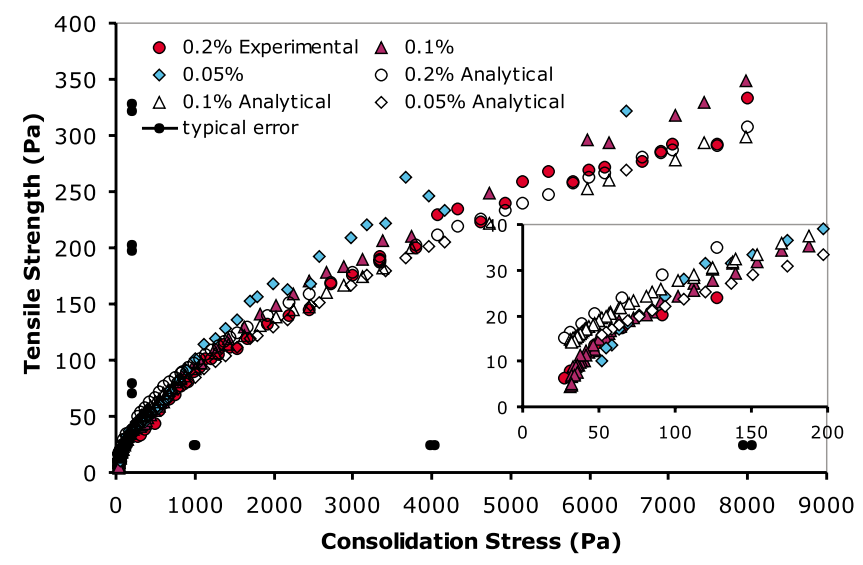

FIG. 10. (Color online) Comparison of experimental with predicted tensile strength. Horizontal and vertical black bars show the typical error in the consolidation stress and the experimental tensile strength.

tested samples since in that range its typical value $(\sim 5 \mathrm{nN})$ is much less than the interparticle compressive force. The external consolidation stress is dominant indicating that the guest particle size has little effect on the contact force when the deformation of contact is fully plastic.

Comparison of the experimental tensile strength (solid symbols) with the predicted values of the tensile strength (open symbols) as a function of consolidation stress is shown in Fig. 10. The predicted values of the tensile strength are very compatible with the experimental results showing the same trend and approximately similar values. This suggests that even under small consolidation, dry-coated particles deform plastically. The inset displays the tensile strength in terms of the consolidation stress less than $200 \mathrm{~Pa}$ and it appears that the analytical tensile strength is greater than the experimental perhaps because the deformation of the host particle surface is elastic or elastic plastic rather than fully plastic.

It is conceivable that above certain consolidation, the plastic deformation of the host particles is big enough to engulf the nanosized guest particles. Once the guest particles

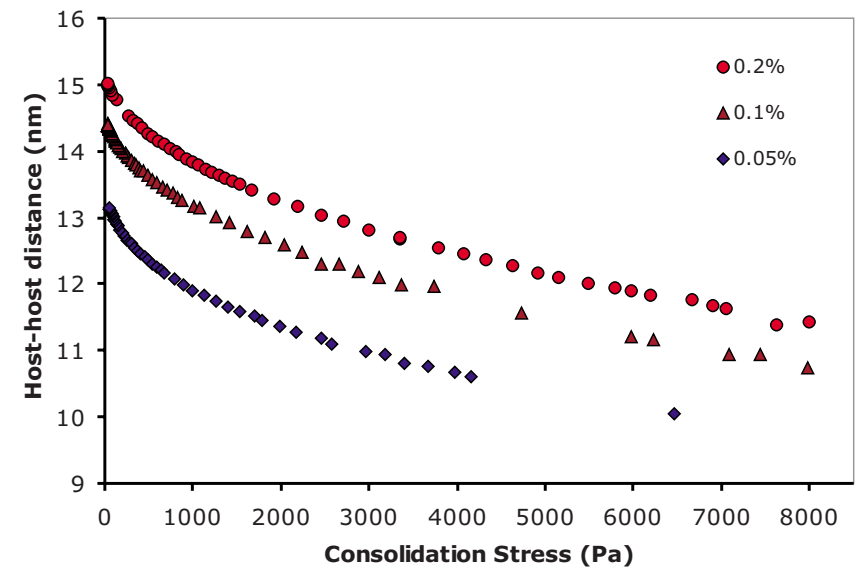

FIG. 11. (Color online) Distance between host surfaces as a function of the consolidation stress for different weight percentages of nanoadditive. 

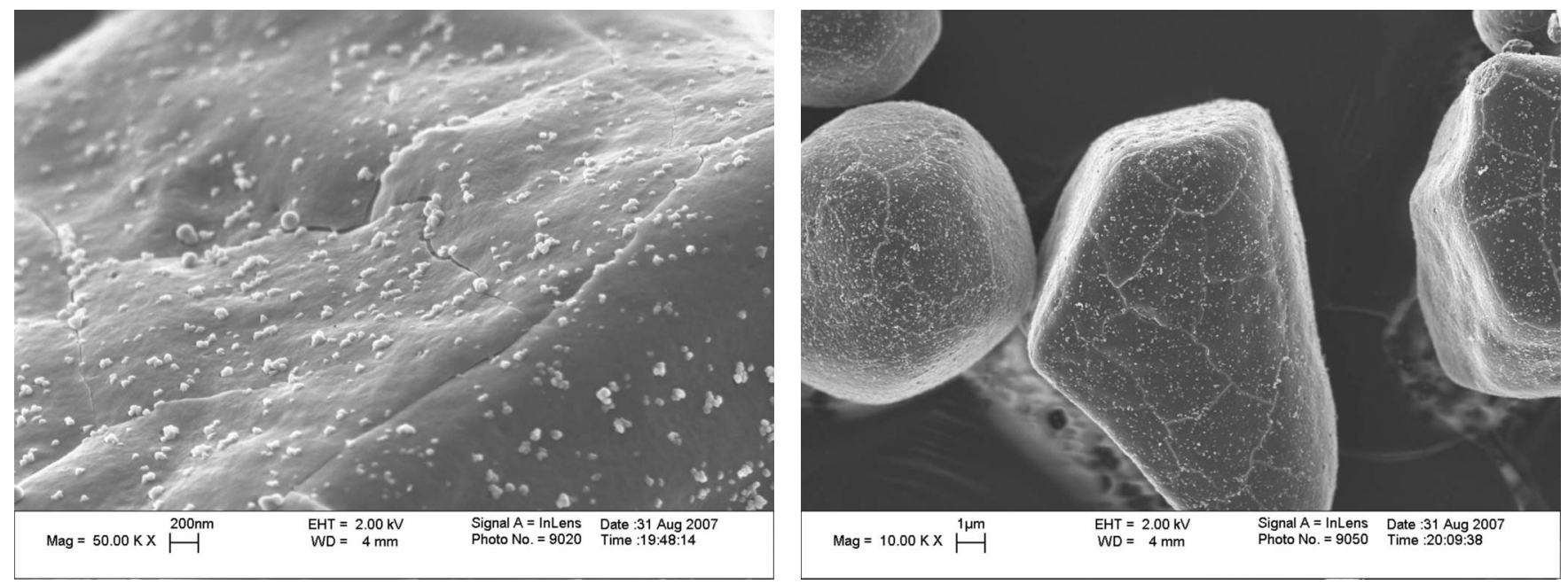

FIG. 12. SEM images of 0.1 wt $\%$ R972 coated cornstarch after being consolidated under $100 \mathrm{KPa}$ for $24 \mathrm{~h}$.

are engulfed in host particle, they would no longer improve flowability or fluidizability since the dry-coated particles would become caked. Rimai et al. [37] pointed out that without considering the external forces, the critical radius of particle $R_{c}$ less than which engulfment would occur when a particle contacts with a flat surface is

$$
R_{c}=2 \gamma_{12} / H,
$$

where, $\gamma_{12}=\gamma_{1}+\gamma_{2}-\sqrt{\gamma_{1} \gamma_{2}}$ is the interfacial surface energy.

It is difficult to calculate analytically the depth of indentation mark on the host particle surface after elastic recovery because the radius of contact area starts to decrease when a load equating the pull-off force is applied, though the radius of the contact area is constant before the load reaches the pull-off force. Here, Eq. (16) is used to estimate the center approach between guest and host particle surface and the distance between host particles is calculated from $\left(L_{0}-2 c\right)$. Host-host distance as a function of consolidation stress corresponding to different coating level is plotted in Fig. 11. As can be seen, the host-host distance decreases with increasing the consolidation stress and larger SAC leads to larger hosthost distances due to better spacing effect of guest particles.

SEM images of 0.1 wt \% R972 coated cornstarch in Fig. 12 illustrate that guest particles remain on the host particle surface after being compressed by $100 \mathrm{Kpa}$ pressure for $24 \mathrm{~h}$. Powders are compacted in a 2.5" Jenike cell and a load is applied through a consolidation bench. Hence consolidations, smaller than this, cannot engulf the guest particles into host particle. This also ensures the improvement on flowability and fluidizability of dry-coated fine powders even under relatively large consolidations.

The ratio of guest-host adhesion force to host-host attractive force defined as the ratio of the first term in Eq. (18) to the second term is presented in Fig. 13 as a function of consolidation stress. It could be seen that for all sparsely coated cornstarch, the guest-host adhesion force is dominant over the host-host adhesion force by 2 orders of magnitude due to the spacing effect of guest particles during elastic recovery. Larger SAC leads to greater force ratio. Thus host- host adhesion force is negligible when estimating the pull-off force between dry-coated fine particles even for consolidations as low as $20 \mathrm{~Pa}$ (the smaller values of the consolidation stress in Fig. 13).

\section{Results for densely coated cornstarch}

Figure 14 shows the experimental tensile strength as a function of the consolidation stress for $0.5 \mathrm{wt} \%$ and 1.0 wt \% R972 coated cornstarch; both of which are densely coated. Figure 14 also displays the predicted tensile strength in terms of the consolidation stress for our two densely coated cornstarch samples. It appears that the predicted tensile stresses are smaller than the experimental results, especially in the case of the 1.0 wt \% coated cornstarch. The measured tensile strength is similar for both $0.5 \mathrm{wt} \%$ and 1.0 wt $\%$ despite the different coating level, while the model predicts a smaller tensile strength for cornstarch $1.0 \mathrm{wt} \%$. It is worth noting that Eq. (20) is only valid when $a / R^{*}$ is less than 0.2. However, even under small consolidation $\left(\sigma_{c}\right.$ $<200 \mathrm{~Pa}$ ) this criterion can be easily reached. $a / R^{*}$ is al-

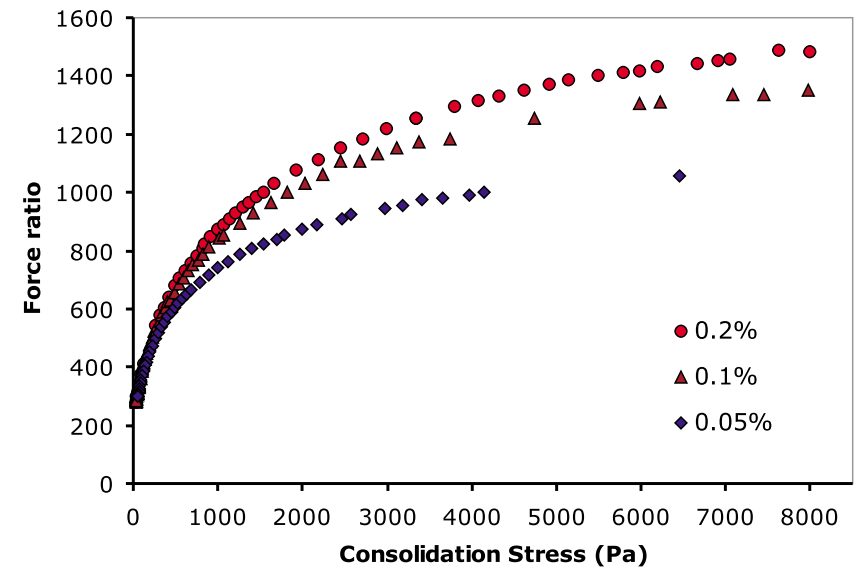

FIG. 13. (Color online) Ratios of guest-host adhesion to hosthost attraction as a function of consolidation stress with different weight percentage of nanoadditives. 


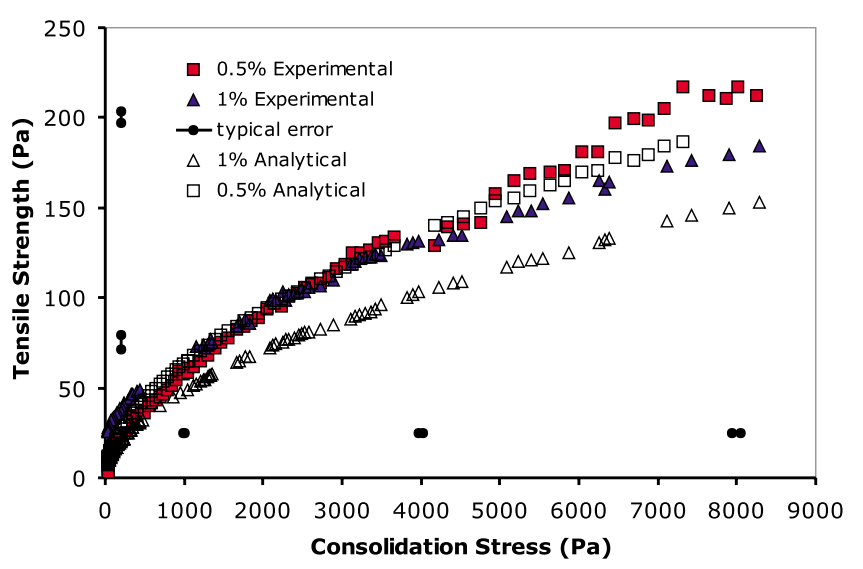

FIG. 14. (Color online) Comparison of experimental with analytical tensile strength for densely coated cornstarch. Horizontal and vertical black bars show the typical error in the consolidation stress and the experimental tensile strength.

ready 0.2 when consolidation stress is $50 \mathrm{~Pa}$ (interparticle compressive force of $27 \mathrm{nN}$ ) according to the calculation using Eq. (19). So, the actual average pressure over the contact area may be smaller than the hardness of the material and the pull-off forces may be greater than those predicted by the model presented here.

Figure 15 depicts typical SEM images of 0.5 wt \% R972 coated cornstarch after $24 \mathrm{~h}$ consolidation with pressure of $100 \mathrm{Kpa}$. Similarly to sparsely coated particles, nanosized silica particles remain on the host particle surface rather than being fully embedded into it though only a small area of total surface is shown in this image.

\section{Sensitivity to the input parameters}

The only parameter that differentiates between the materials discussed in Sec. II E is the SAC. The SAC is calculated from Eq. (7) using the densities of the particles, the diameter of the additive particle (all of them quantities that can be measured with a good degree of accuracy), the weight ratio of the additive added when the material is treated, and the typical diameter $D$ of the asperities on the surface of the host particles. This last quantity has not been measured, since to obtain a value for it would have required recording the topography of the host particles. While it is possible to obtain the topography of $15 \mu \mathrm{m}$ particles by means of atomic force microscopy or optical confocal microscopy, the amount of work required to analyze a significant number of particles to get a statistically meaningful result makes such an undertaking out of the scope of this work, apart from the fact that the surface of the particles would show irregularities with sizes up to comparable to the particle size and down to the scale of the nanometer and to get a single representative value of the asperity size $D$ out of the real topography of the particles would not be an easy and uncontroversial task. For these reasons, we have decided to use a reasonable guess for the value of $D(1 \mu \mathrm{m}$, as stated in Sec. II C). Therefore, the values of the SAC have considerable degree of uncertainty; but as it will become apparent after this discussion, the uncertainty in the value of the SAC does not have a large effect in the predictions of the model presented in Secs. IV B and IV C.

The SAC intervenes in the model by determining if a material is sparsely or densely coated. Note that the boundary between both types of coating is given by Eq. (10), where the asperity size $D$ does not intervene. Therefore this boundary is unaffected by the uncertainty in the value of $D$. Additionally, for sparsely coated materials, the SAC determines the initial separation $L_{0}$ between host surfaces [see Eq. (8) and Fig. 11] and for densely coated materials the angle of contact $\theta$ between guest particles [see Eq. (22) and its accompanying discussion].

For the sparsely coated cornstarch, the change in the SAC does not cause a major change in the separation between host surfaces. For example, in Fig. 11, it is seen that the separation between host surfaces increases only about $1 \mathrm{~nm}$ in the whole range of consolidation stresses studied when the SAC increases from a $6.9 \%$ [the value yielded by Eq. (7) for a weight ratio of $0.05 \%$ ] to a $27.5 \%$ (equation for a weight ratio of $0.2 \%$ ). This change in the distance $L$ does not affect significantly either the compressive force between the particles or the predicted tensile strength (see Figs. 9 and 10)
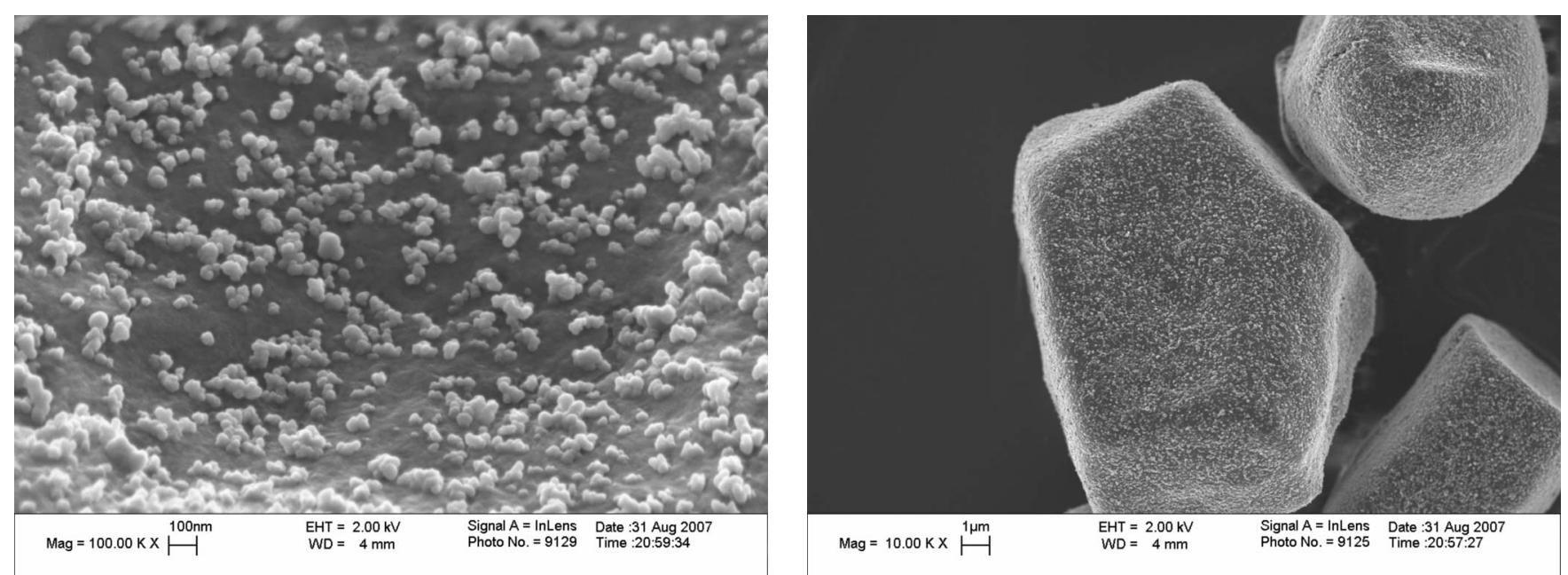

FIG. 15. SEM. Images of 0.5 wt \% R972 coated cornstarch consolidated by $100 \mathrm{KPa}$ pressure for $24 \mathrm{~h}$. 


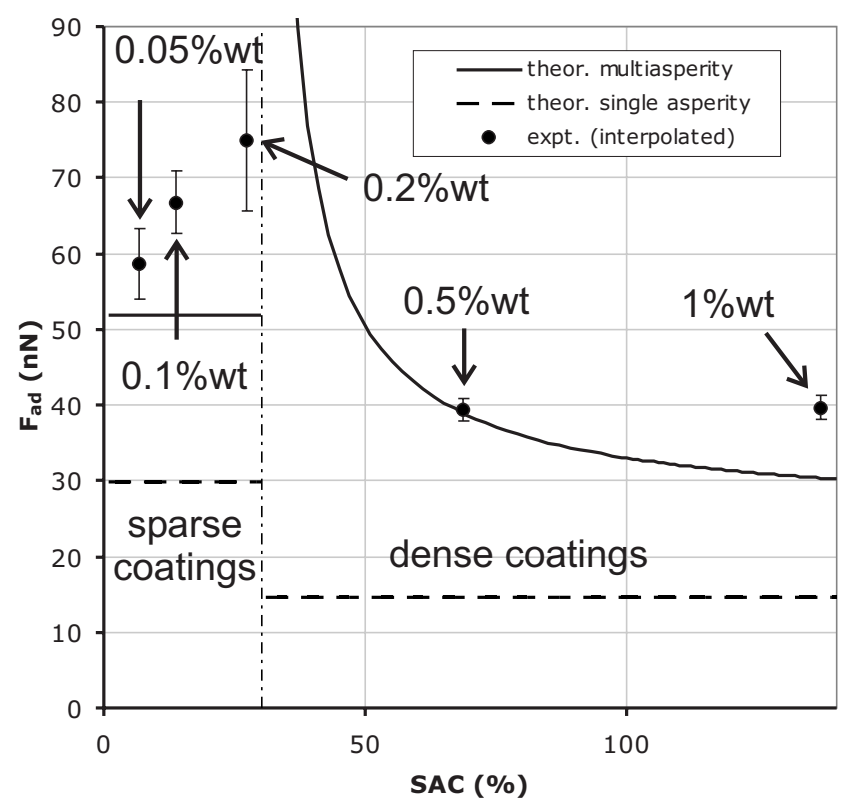

FIG. 16. Values of the pull-off force for the cornstarch samples vs their SAC and predictions of the models in Sec. III. The compressive interparticle force $F_{\text {on }}$ is fixed at $1000 \mathrm{nN}$. The data point of each material has been found by interpolating the results of Eq. (24) when applied to the solid fraction in Fig. 5 and the tensile strength in Fig. 6. Error bars represent three times the expected standard deviation from the central value at $F_{\text {on }}=1000 \mathrm{nN}$ given by the interpolation.

except at the smallest values of the compressive force because the attractive force between host surfaces, which depends on the distance $L$, is always much smaller than the attractive force between guest and host particles (see Fig. 13). The consequences of this fact are twofold. First, the pull-off force $F_{a d}$ predicted by the model for sparsely coated cornstarch does not depend significantly on the SAC, as it can be seen in Fig. 16, where we present the dependence of the predicted pull-off force $F_{a d}$ with the SAC for an interparticle compressive force $F_{\text {on }}=1000 \mathrm{nN}$; second, the pull-off force $F_{a d}$ for sparsely coated cornstarch does not change appreciably by a change in the typical asperity size $D$, as the second term in Eq. (18) is always much smaller that the first term representing the contact adhesion between guest and host (see Fig. 13).

In the case of the pull-off force $F_{a d}$ for densely coated cornstarch [Eq. (23)], we have decided to neglect the van der Waals interaction between host surfaces because their separation is even larger than in the case of sparse coating. Hence the typical asperity size $D$ does not directly influence the predicted pull-off force $F_{a d}$ for dense coating but it does influence it indirectly, as the angle of contact $\theta$ does vary significantly with the SAC. In consequence, the pull-off force between the particles predicted by Eq. (23) does depend on the SAC: $F_{a d}$ is a monotonically decreasing function with increasing SAC, with $F_{a d}$ decreasing rapidly when the value of the SAC is close to the limiting value between sparse and dense coatings $C_{S A}^{\text {guest-guest }}$ and a gentler decrease for larger values of SAC.

In conclusion, the indeterminacy in the value of the SAC originated by the indeterminacy in the typical asperity size $D$ does not cause a large indeterminacy in the predicted value of the pull-off force $F_{a d}$ or the predicted tensile strength as long as the real SAC of the materials is not close to the limiting value $C_{S A}^{\text {guest-guest }}$ between sparse and dense coatings.

Note that in Fig. 16 the predictions for sparse and dense coatings do not converge to the same value at $C_{S A}^{\text {guest-guest }}$. The reason is that for values slightly larger than $C_{S A}^{\text {guest-guest }}$, the angle of contact $\theta$ in Eq. (22) tends to $\pi / 2$ and the normal contact load $F_{c}$ diverges. The cause of the divergence is that the physical mechanism of separation between guest particles outlined in Sec. III B is not correct for angles of contact close to $\pi / 2$ (alt SAC close to $C_{S A}^{\text {guest-guest }}$ ). In this situation, the geometrical arrangement of guest particles is such that their contact is better described by a frictional contact broken by peeling rather than by a normal contact broken by brittle separation, which was the picture described in Sec. III B. Thus, a different model based on the frictional contact between guest particles and noncontact van der Waals interaction between host and guest particles would be needed in the range of SAC slightly larger than $C_{S A}^{\text {guest-guest }}$ to make the predictions of the models for sparse and dense coatings join smoothly at $C_{S A}^{\text {guest-guest }}$. These details of the transition between sparse and dense coatings would not have been uncovered had we considered only single asperity contacts between particles, as done in a previous model [24]. In a single asperity model, the results for the pull-off force experience a sharp change when the SAC crosses the boundary between dense and sparse coatings, as it has also been shown in Fig. 16. Apart from this qualitative difference, the predicted values of the pull-off force are lower for the single asperity model than those obtained in the multiasperity model presented in this paper because in the multiasperity model the total pull-off force is the sum of the contributions of three single asperity contacts; hence the present model more closely matches the experimental results.

Finally, in Fig. 16 we have extended the prediction of the densely coated model for values of the SAC larger than $100 \%$ since Eq. (7) using $D=1 \mu \mathrm{m}$ predicts $C_{S A}=137 \%$ for the material treated with 1 wt $\%$ of silica R972, in agreement with Fig. 2(e), where it can be seen that the silica particles form more than a monolayer on the surface of this material.

Another parameter with a large degree of indeterminancy is the work of adhesion. For example, in Ref. [38], three values for the surface energy $\gamma$ of silica are quoted: $\gamma=14 \mathrm{~mJ} / \mathrm{m}^{2}, 25 \mathrm{~mJ} / \mathrm{m}^{2}$, and $40 \mathrm{~mJ} / \mathrm{m}^{2}$ (in Table I we have used the central value of those given in Ref. [38]), giving a spread of about $48 \%$ of the central value $(\gamma$ $=27 \mathrm{~mJ} / \mathrm{m}^{2}$ ). Although the surface energy of cornstarch has not been measured experimentally: in fact, the value listed in Table I corresponds to the typical value for polymers listed in Ref. [36]. Based on the uncertainty in the value of the surface energy of silica, we will assume that a typical value in the uncertainties of the surface energy and the work of adhesion is a $50 \%$. Figure 17 shows the pull-off force estimated from the values of the solid fraction and the tensile strength shown in Figs. 5 and 6 and the predictions of the models for sparse and dense coatings using a work of adhesion $\Delta \gamma$ $=71 \mathrm{~mJ} / \mathrm{m}^{2}$ for the work of adhesion of the silica-cornstarch surface $\left(\Delta \gamma=\sqrt{\gamma_{1} \gamma_{2}}\right)$ and $\Delta \gamma=50 \mathrm{~mJ} / \mathrm{m}^{2}$ for the work of 


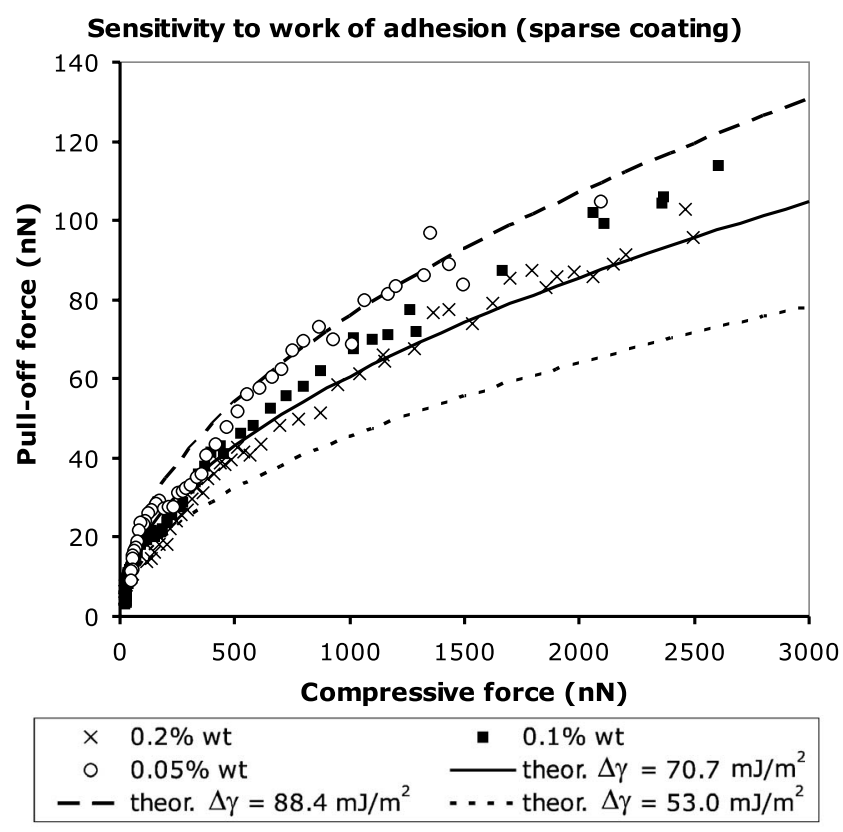

Sensitivity to work of adhesion (dense coating)

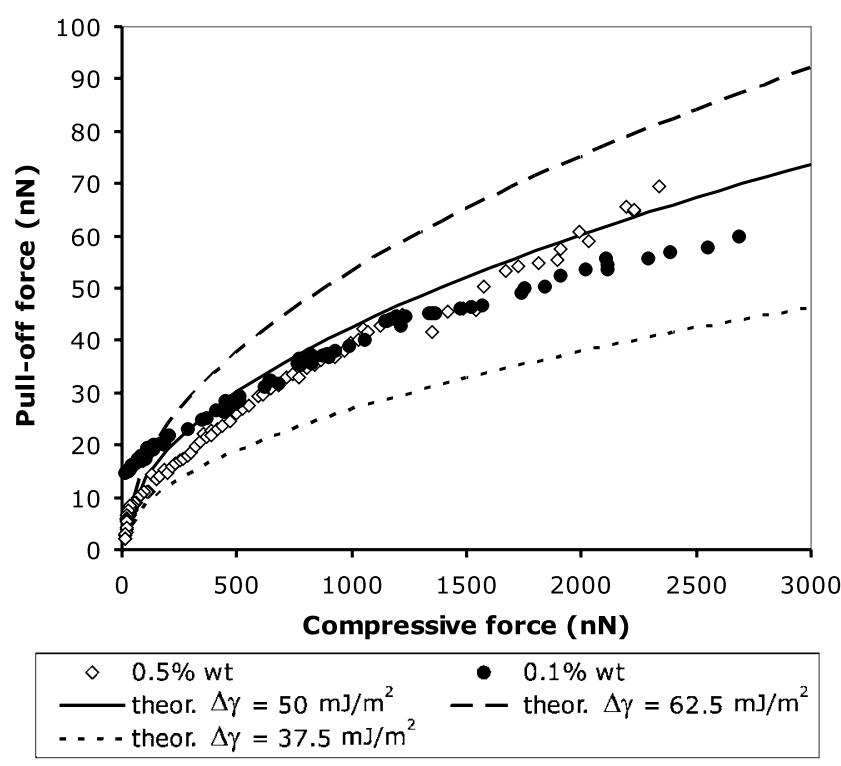

FIG. 17. Effect of the work of adhesion on the predicted interparticle pull-off force. The results of the dense coating model correspond to surface area coverage of $60 \%$.

adhesion of silica and also for $25 \%$ of those values. While the predictions of the models do not exactly match the estimated pull-off force, it is seen from Fig. 17 that the difference between estimation and prediction may be attributed to the uncertainty in the values of the work of adhesion, since the estimated values lay within the band limited by the upper and lower values of the work of adhesion used in Fig. 17.

In the case of the hardness $H$, the values reported in the literature for fused silica range from 4.5 to $9.2 \mathrm{GPa}$ (fused silica was chosen rather than other forms or silica based on the assumption that silica nanoparticles have the amorphous structure of fused silica). Differences in the values arise from the different experimental procedures (mainly the shape of

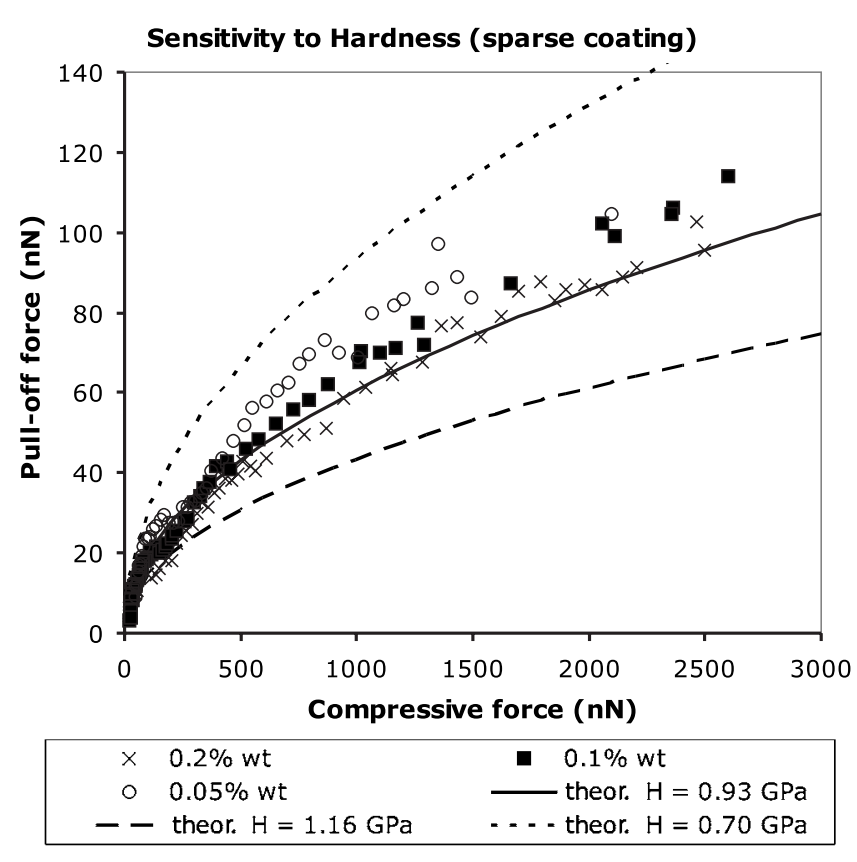

Sensitivity to Hardness (dense coating)

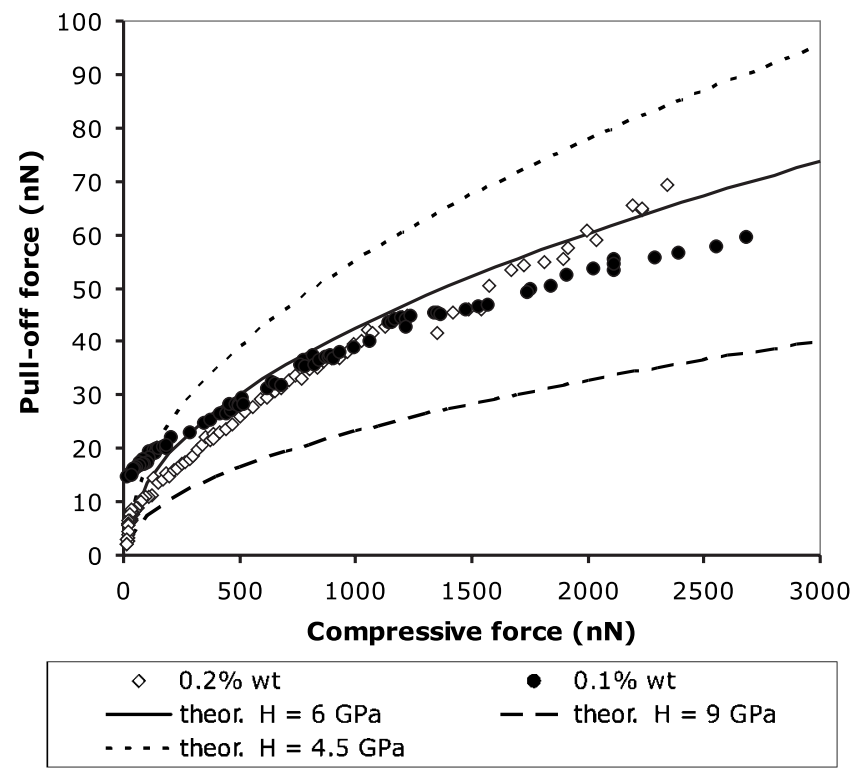

FIG. 18. Effect of hardness on the prediction of the interparticle pull-off force by the sparse coating (top) and dense coating (bottom) models. The results of the dense coating model correspond to a surface area coverage of $60 \%$.

the microscopic indenter) used to measure the hardness in the microscopic scale: Knoop tests (elongated indenters) throw values ranging from 4.5 [39] to 5.5-9.0 GPa [40] while Berkovich tests (symmetric indenters) throw values close to $9 \mathrm{GPa}$ (8.6 in Ref. [41] and 9.2 in Ref. [40]). The results of the dense coating model for the value listed in Table I and for the limiting values of the range found in the literature are shown in Fig. 18, where it is seen that the pull-off force for densely coated cornstarch estimated from the values of the solid fraction and the tensile strength lay close to the theoretical predictions using the central value of the hardness of fused silica given in the literature. For 
sparsely coated cornstarch, we could not find other values of the hardness in the literature other than that listed in Ref. [42] $H=0.93 \mathrm{GPa}$, which yields a theoretical prediction for the pull-off force somewhat below the estimated values. However, all the data points lay within a band limited by the predictions using $25 \%$ of the value listed in the literature $(H=0.93 \mathrm{GPa})$.

Regarding the effect of the effective Young's modulus $E^{*}$ on the predicted pull-off force, it is not necessary to make a similar figure as Fig. 18, as in Eqs. (18) and (23) the pull-off force is proportional to the product $\Delta \gamma E^{*}$ (there is a dependency with $2 \pi \Delta \gamma R^{*}$ included in the contact force $F_{c}$, but this term in $F_{c}$ can be neglected in most of the range of values of the interparticle compressive force $F_{\text {on }}$ ). Due to this fact, the same relative changes in $\Delta \gamma$ and $E^{*}$ cause the same relative changes in $F_{a d}$. Apart from this, all references coincide in giving a Young's modulus close to $70 \mathrm{GPa}$ for silica $[25,40,41,43]$, which means that the only significant source of experimental error in the value of the reduced Young's modulus $E^{*}$ arises from the Young's modulus of cornstarch.

\section{CONCLUSIONS}

The interparticle pull-off force for sparsely and densely dry-coated cohesive powders has been investigated theoretically and experimentally. A three-dimensional adhesion force model has been proposed to predict the pull-off force (defined as the adhesion force at the moment when the two contacting surfaces are separated) by taking into account the fully plastic deformation on the contact area and SAC of nanosized guest particles. The pull-off forces are estimated from experimentally measured bulk tensile strength and solid fraction of dry-coated fine particles.

A transition criterion between sparse coating and dense coating is proposed to determine the coating type based on the value of the SAC. For both coating types, the pull-off force increases nonlinearly with increasing consolidation stress.

The experimental and analytical results indicate that pulloff forces are significantly different between sparsely coated particles and densely coated particles since the contact hardness of guest-guest contacts is much larger than the contact hardness of guest-host contacts. Nevertheless, within a specific coating coverage type, e.g., sparse coating or dense coating, SAC does not have major effect on the pull-off forces since the predominant type of contact is the same in a given coating coverage type.

The deformation of the cornstarch surface increases with the consolidation force and decreases with the SAC. Under the consolidation less than $100 \mathrm{KPa}$, the deformation of the cornstarch particle is not large enough to fully embed the nanosized silica inside the cornstarch particle. SEM images also verified these predictions.

\section{ACKNOWLEDGMENTS}

Financial support from the NSF (Grant No. EEC0540855) for part of this work is gratefully acknowledged by Y.C., R.D., and M.A.S.Q. The Seville group acknowledges the Spanish Government Agency Ministerio de Ciencia y Tecnologia (Contract No. FIS2006-03645) and the Junta de Andalucia (Contract No. FQM 421).
[1] D. Geldart, Powder Technol. 7, 285 (1973).

[2] A. B. Yu, C. L. Feng, R. P. Zou, and R. Y. Yang, Powder Technol. 130, 70 (2003).

[3] R. Pfeffer, R. Dave, D. Wei, and M. Ramlakhan, Powder Technol. 117, 40 (2001).

[4] J. Yang, A. Sliva, A. Banerjee, R. Dave, and R. Pfeffer, Powder Technol. 158, 21 (2005).

[5] Y. Chen, J. Yang, R. N. Dave, and R. Pfeffer, AIChE J. 54, 104 (2008).

[6] C. Lauga, J. Chaouki, D. Klvana, and C. Chavarie, Powder Technol. 65, 461 (1991).

[7] H. Rumpf, Particle Technology (Chapman and Hall, London, 1990).

[8] H. Xie, Powder Technol. 94, 99 (1997).

[9] A. M. Hollenbach, A. M. Peleg, and R. Rufner, Powder Technol. 35, 51 (1983).

[10] H. O. Kono, C. Hung, and M. Xi, AIChE Symp. Ser. 85, 44 (1989).

[11] A. Dutta and L. V. Dullea, AIChE Symp. Ser. 86, 26 (1990).

[12] K. L. Johnson, K. Kendall, and A. D. Roberts, Proc. R. Soc. London, Ser. A 324, 301 (1971).

[13] B. V. Derjaguin, V. M. Muller, and Y. P. Toporov, J. Colloid Interface Sci. 53, 314 (1975).

[14] F. Podczeck, Particle-particle Adhesion in Pharmaceutical Powder Handling (Imperial College, London, 1998).
[15] F. Podczeck and J. M. Newton, J. Appl. Phys. 79, 1458 (1996).

[16] R. Mei, H. Shang, J. F. Klausner, and E. Kallman, Kona 15, 132 (1997).

[17] Y. I. Rabinovich, J. J. Adler, A. Ata, R. K. Singh, and B. M. Moudgil, J. Colloid Interface Sci. 232, 10 (2000).

[18] Y. I. Rabinovich, J. J. Adler, A. Ata, R. K. Singh, and B. M. Moudgil, J. Colloid Interface Sci. 232, 17 (2000).

[19] Q. Li, V. Rudolph, and W. Peukert, Powder Technol. 161, 248 (2006).

[20] J. Tomas, Part. Sci. Technol. 19, 95 (2001).

[21] A. Castellanos, Adv. Phys. 54, 263 (2005).

[22] A. Castellanos, J. M. Valverde, and M. A. S. Quintanilla, Kona 22, 1 (2004).

[23] J. M. Valverde, A. Castellanos, A. Ramos, A. T. Pérez, M. A. Morgan, and K. Watson, Rev. Sci. Instrum. 71, 2791 (2000).

[24] M. A. S. Quintanilla, A. Castellanos, and J. M. Valverde, Phys. Rev. E 64, 031301 (2001).

[25] F. Restagno, J. Crassous, C. Cottin-Bizonne, and E. Charlaix, Phys. Rev. E 65, 042301 (2002).

[26] J. Q. Feng, J. Colloid Interface Sci. 238, 318 (2001).

[27] L. Kogut and I. Etsion, J. Colloid Interface Sci. 261, 372 (2003).

[28] K. L. Johnson, in Adhesion at the Contact of Solids: Theoretical and Applied Mechanics, edited by W. T. Koiter (NorthHolland, Amsterdam, 1976), p. 133-143. 
[29] D. Maugis and H. M. Pollock, Acta Metall. 32, 1323 (1984).

[30] M. A. S. Quintanilla, J. M. Valverde, and A. Castellanos, AIChE J. 52, 1715 (2006).

[31] J. M. Valverde, A. Castellanos, and P. K. Watson, Powder Technol. 118, 236 (2001).

[32] D. Tabor, J. Colloid Interface Sci. 58, 2 (1977).

[33] L. Massimilla and G. Donsi, Powder Technol. 15, 253 (1976).

[34] C. Thornton and Z. Ning, Powder Technol. 99, 154 (1998).

[35] S. D. Mesarovic and N. A. Fleck, Int. J. Solids Struct. 37, 7071 (2000).

[36] S. D. Mesarovic and K. L. Johnson, J. Mech. Phys. Solids 48, 2009 (2000).
[37] D. S. Rimai, L. P. Demejo, and R. C. Bowen, J. Adhes. Sci. Technol. 8, 1333 (1994).

[38] L.-O. Heim, J. Blum, M. Preuss, and H.-J. Butt, Phys. Rev. Lett. 83, 3328 (1999).

[39] L. Riester, P. J. Blau, E. Lara-Curzio, and K. Breder, Thin Solid Films 377-378, 635 (2000).

[40] L. Zheng, A. W. Schmid, and J. C. Lambropoulos, J. Mater. Sci. 42, 191 (2007).

[41] W. C. Oliver and G. M. Pharr, J. Mater. Res. 7, 1564 (1992).

[42] P. Paronen and M. Juslin, J. Pharm. Pharmacol. 35, 627 (1983).

[43] http://www.accuratus.com/fused.html 